\title{
Human butyrylcholinesterase polymorphism: Molecular modeling
}

\author{
S. Lushchekina ${ }^{\mathrm{a}, *}$, H. Delacour ${ }^{\mathrm{b}}$, O. Lockridge ${ }^{\mathrm{c}}$ and P. Masson ${ }^{\mathrm{c}, \mathrm{d}}$ \\ ${ }^{a}$ Emanuel Institute of Biochemical Physics, Moscow, Russia \\ 'Bégin Military Teaching Hospital, Saint Mandé, France \\ 'University of Nebraska Medical Center, Omaha, USA \\ dKazan Federal University, Kazan, Russia \\ *Corresponding author. E-mail: sofya.lushchekina@gmail.com
}

BACKGROUND: Prolonged apnoea following injection of ester-containing myoralaxants was first described in 1953. Because a large part of administered succinylcholine is shortly hydrolyzed by plasma butyrylcholinesterase $(\mathrm{BChE})$ under normal conditions, prolonged apnoea was attributed to deficiency in $\mathrm{BChE}$. It was found that $\mathrm{BChE}$ deficiency was due to genetic variations. Human $\mathrm{BChE}$ gene shows a large polyallelism. About 75 natural mutations of the $B C H E$ gene have been documented so far [1]. Most of them cause alteration in BChE activity through point mutation effect on catalytic activity. Frame shifts and stop codons may also affect expression, or cause truncations in the sequence.

OBJECTIVE: Recently, two novel BChE "silent" variants, Val204Asp [2] and Ala34Val [3], causing prolonged neuromuscular block after administration of mivacurium, were discovered. Mutations were genetically and kinetically characterized. The aim of the current study was to understand how these mutations determine "silent" phenotype.

METHODS: Molecular dynamics studies were carried out with NAMD 2.9 software at the Lomonosov supercomputer. Charmm 36 force field was used, periodical boundary conditions, $1 \mathrm{~atm}$ pressure, $298 \mathrm{~K}$. $100 \mathrm{~ns}$ molecular dynamics runs were performed for the wild-type BChE and its mutants Val204Asp and Ala34Val.

RESULTS: Unlike wild-type $\mathrm{BChE}$, which retained its operative catalytic triad through the whole MD simulation, the catalytic triad of mutants was disrupted, making chemical step impossible. Val204Asp mutation leads to reorganization of hydrogen bonding network around the catalytic triad, which in turn increases the distance between catalytic residue main chains. Mutation Ala34Val, located on the protein surface, leads to increased fluctuations in the $\Omega$-loop and subsequent disruption of the gorge structure, including disruption of the catalytic triad and formation of new hydrogen bonds involving catalytic center residues.

CONCLUSIONS: Comparative study of the "silent" Ala328Asp mutant and the catalytically active mutant Ala328Cys shows that MD approach can discriminate between the differential effects of point mutations at a same position.

Keywords: Genetic, polymorphism, butyrylcholinesterase, molecular modeling, allelozymes 


\section{Conflict of interest statement: None.}

\section{References}

[1] Lockridge, O. Review of human butyrylcholinesterase structure, function, genetic variants, history of use in the clinic, and potential therapeutic uses. Pharmacol Ther. 2015;148:34-46. doi: 10.1016/j.pharmthera.2014.11.011. Epub 2014 Nov 20.

[2] Delacour, H., Lushchekina, S. et al. Characterization of a Novel BCHE "Silent" Allele: Point Mutation (p.Val204Asp) Causes Loss of Activity and Prolonged Apnea with Suxamethonium. PLoS One. 2014; 9(7): Published online 2014 Jul 23. doi: 10.1371/journal.pone.0101552

[3] Delacour, H., Lushchekina, S. et al. Characterization of a novel butyrylcholinesterase point mutation (p.Ala34Val), "silent" with mivacurium. Biochem Pharmacol. 2014;92(3):476-83. doi: 10.1016/j.bcp.2014.09.014. Epub 2014 Sep 28. 